\title{
Refining of 2, 2-dimethylolpropionaldehyde and Recovering of the Excessive Raw Material
}

\author{
Sai-Jian SHI ${ }^{a}$, Xin HUANG ${ }^{b}$, Xiao-Yan $\mathrm{CAO}^{\mathrm{c}}$, Zheng-Gui GU ${ }^{\mathrm{d},{ }^{*}}$ \\ College of Chemistry and Materials Science, Nanjing Normal University, Nanjing 210023, China \\ a18251370276@163.com, b15952003148@163.com, '18251370670@163.com, \\ dguzhenggui@njnu.edu.cn \\ ${ }^{*}$ Corresponding author
}

Keywords: Continuous Side-distillation, Aspen Plus, Refining and Recovery, Dimethylolpropionaldehyde, Methanol and Propylaldehyde, Orthogonal Experiment.

\begin{abstract}
This study establishes a method of refining the 2, 2-dimethylolpropionaldehyde and recovering the methanol and propylaldehyde by continuous side-distillation and distillation, the process was simulated by Aspen Plus software. In addition, the effects of the theoretical stage numbers, feed stage, side-stream stage, reflux ratio and side-stream ratio are studied and optimized to obtain the optimal operating conditions. Under the optimal operating conditions, the mass fraction and yield of the 2,2-dimethylolpropionaldehyde in side-stream reach $99.01 \%$ and $99.91 \%$ respectively. After recycling the mixture from the side-distillation tower top, the total mass fraction of the methanol and propylaldehyde reaches $99.39 \%$, and the yield of them reaches $99.96 \%$ and $99.99 \%$ respectively, meanwhile the mass fraction of the water reaches $99.99 \%$.
\end{abstract}

\section{Introduction}

2,2-dimethylolpropionaldehyde is an important organic synthesis intermediates[1]. And the dimethylolpropionic acid (DMPA) oxidized from the 2,2-dimethylolpropionaldehyde directly is a kind of the fine chemical products. What's more, DMPA also is the important raw material of the waterborne polyurethane materials. Meanwhile it is used as an additive in the production of the leather chemical material, adhesive and photosensitive resin.

In this study, the 2,2-dimethylolpropionaldehyde is synthesized by the methanol and propylaldehyde and the proportion of them is $2.1: 1$ (mol). When the temperature is $105^{\circ} \mathrm{C}$, the raw material is fed by the reciprocating metering pump with no pulse to the reactor, and the feed rate of them is $0.43: 0.2$ (the catalyst triethylamine is mixed with the methanol). And the product contains a large number of the methanol, water and a little propylaldehyde.

This study introduces a method of refining the 2,2-dimethylolpropionaldehyde and recovering the methanol and propylaldehyde by combining continuous side-distillation with distillation. In addition, the process of the refining and recovering is simulated by the Aspen Plus Simulation software. And different operating conditions affecting the results of the separation are studied to provide the theoretical basis for the further study.

\section{Process Design}

This separation simulation needs two towers, one tower for continuous side-distillation and a second tower for the excessive raw material recovery. The mixture is fed to the first tower, where the high boiling components are separated from the tower bottom. And the top distillate is fed to the second tower to be recovered. In addition, the 2,2-dimethylolpropionaldehyde is separated from the side-stream. Aspen model of this separation process is presented in Figure 1. And the NRTL[2] model is used in this simulation. 


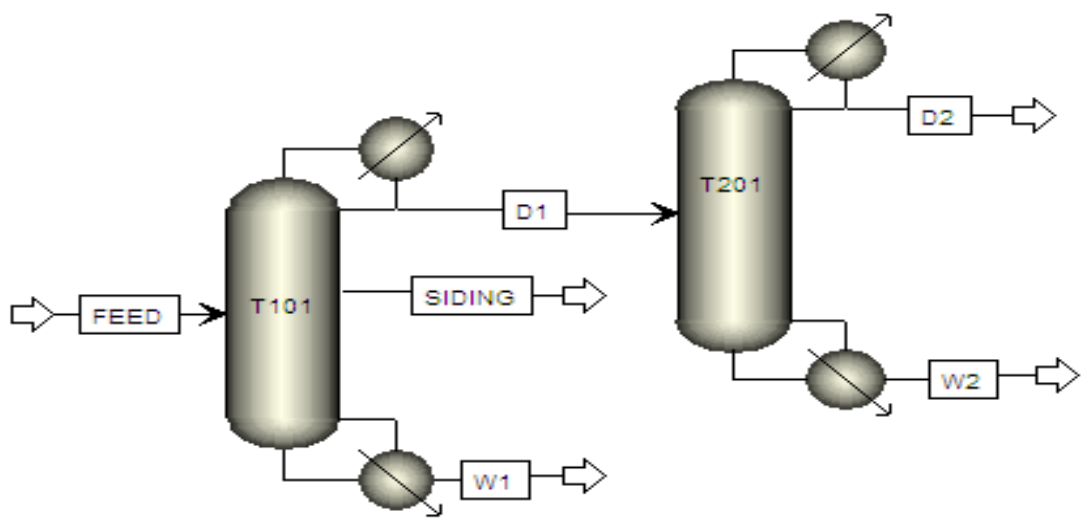

Fig.1 Aspen model of the continuous side-distillation and distillation process 1 Continuous side-distillation column(T101)2 Continuous distillation column(T201)

\section{Simulation}

The simulation provides a theoretical basis for the continuous side-distillation of the mixture. In order to optimize the operating conditions, five factors are studied through the sensitivity analysis in software, including the theoretical stage number, feed stage, side-stream stage, reflux ratio and side-stream ratio.

\section{The Theoretical Stage Number}

Figure 2 shows the influence of the number of theoretical stages on the distillate mass fraction and yield $[3,4]$. As can be seen, when the feed position at stage 11 , side-stream position at stage 15 , reflux ratio at 3.0 and side-stream ratio at 0.06 , the mass fraction and yield of the 2,2-dimethylolpropionaldehyde rise as the increasing of the number of theoretical stages in side-stream. And from figure 2, is also possible to observe that with a number of stages greater than 24 the mass fraction and yield of the distillate do not change significantly. Considering the influence of the investment and operation cost of the tower by the increasing of the number of theoretical stages, so the feasible number of theoretical stages is 24 .

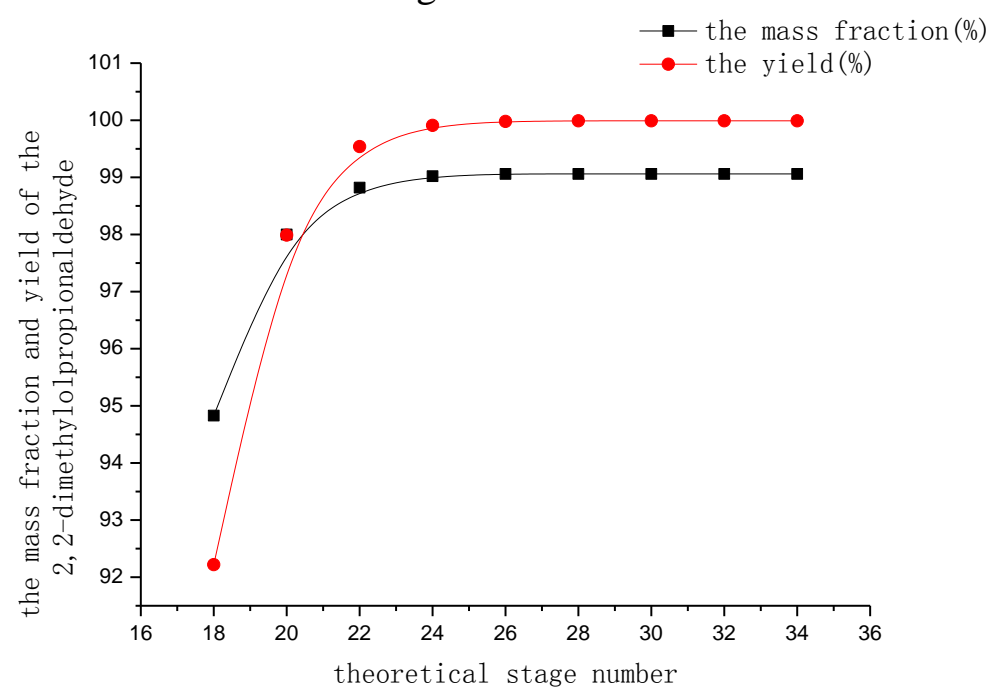

Fig. 2 The influence of the theoretical stage number

\section{Feed Stage}

Figure 3 shows the results of the feed stage analysis. The number of theoretical stages is kept constant (24). As we can see that the mass fraction and yield of the 2,2-dimethylolpropionaldehyde in side-stream rise when the mixture is fed between 2 and 11. And at feed stage 11 is obtained the greatest distillate compositions and yield. It is also possible to observe that the mass fraction and 
yield decline when the feed stage is greater than 11. Hence, a feasible feed position is at stage 11.

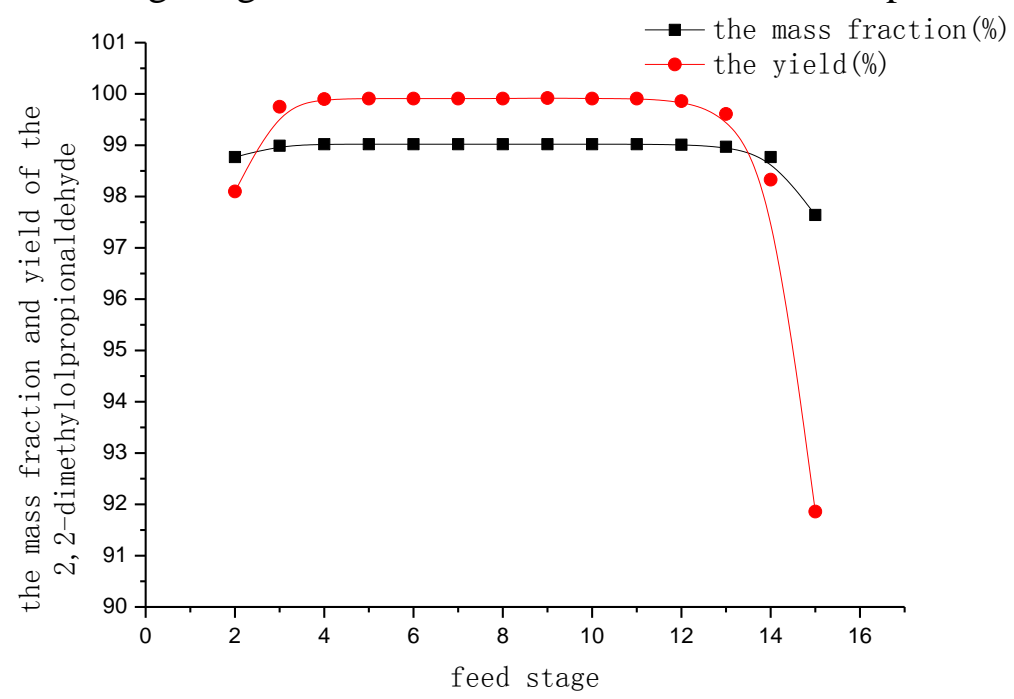

Fig. 3 The influence of the feed stage

\section{Side-Stream Stage}

The side-stream stage effect on the distillate 2,2-dimethylolpropionaldehyde is presented in Figure 4. The feed stage is maintained at 11, whilst the side-stream varies from 7 to 19 . It can be seen from figures that mass fraction and yield of 2,2-dimethylolpropionaldehyde do not change largely at first. However, when side-stream varies from 13 to 15 , the distillate mass fraction and yield in side-stream rise obviously. In addition, the highest distillate compositions and yield are achieved at side-stream stage 15 , and then this is a feasible side-stream stage.

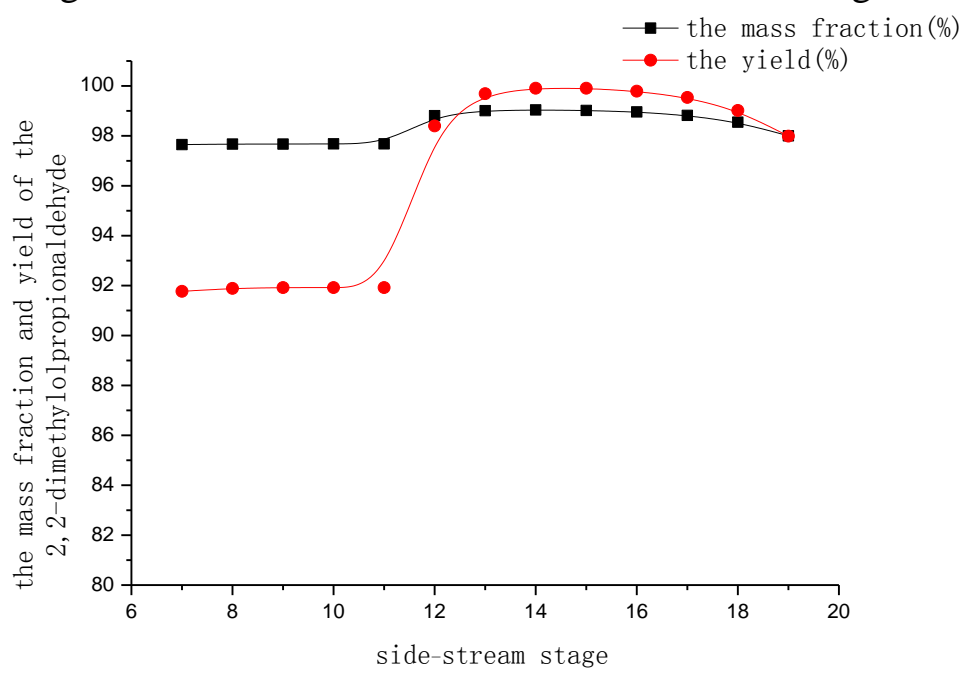

Fig. 4 The influence of the side-stream stage

\section{Reflux Ratio}

Figure 5 presents the influence of the reflux ratio on the distillate mass fraction and yield. And the side-stream stage is kept constant (15). It is observed that the mass fraction and yield of the 2,2-dimethylolpropionaldehyde in side-stream rise as the increasing of the reflux ratio. But when the reflux ratio varies in the range of 1.0 to 3.0, the distillate compositions change largely. At reflux ratio between 3.0 and 5.0 are reached the best results, with a difference them no higher than 0.03 . However, the reflux ratio has an important effect on the column energy consumption, because of the greater reflux ratio, the more reboiler and condenser duties[5]. From the above analysis, at a reflux ratio of 3.0 is feasible. 


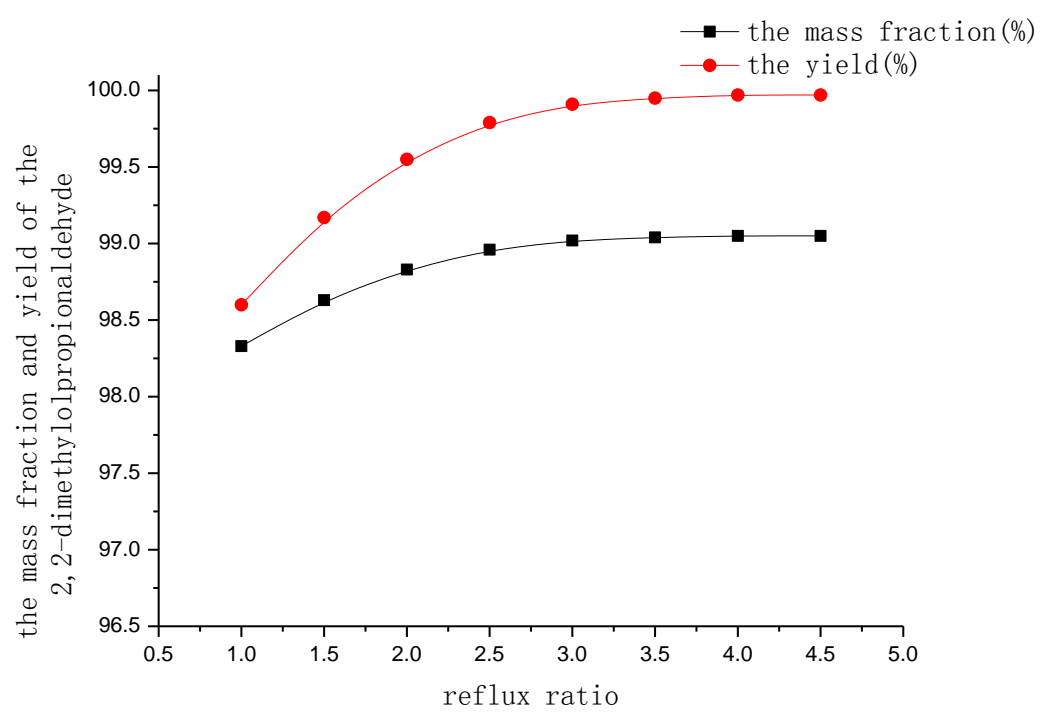

Fig.5 The influence of the reflux ratio

\section{Side-Stream Ratio}

The side-stream ratio effect on the distillate mass fraction and yield is shown in the Figure 6 at the reflux ratio of 3.0. As shown in Figure 6, the mass fraction of the 2,2-dimethylolpropionaldehyde does not change obviously when the side-stream ratio is between 0.02 and 0.06 , and then it decreases when side-stream ratio is greater than 0.06 . Nevertheless, the yield rises as the increasing of the side-stream ratio[6]. And it is almost constant when the side-stream ratio varies from 0.06 to 0.09 . From these results, at side-stream ratio of 0.06 , the mass fraction and yield are both the best, so the feasible side-stream ratio is 0.06 .

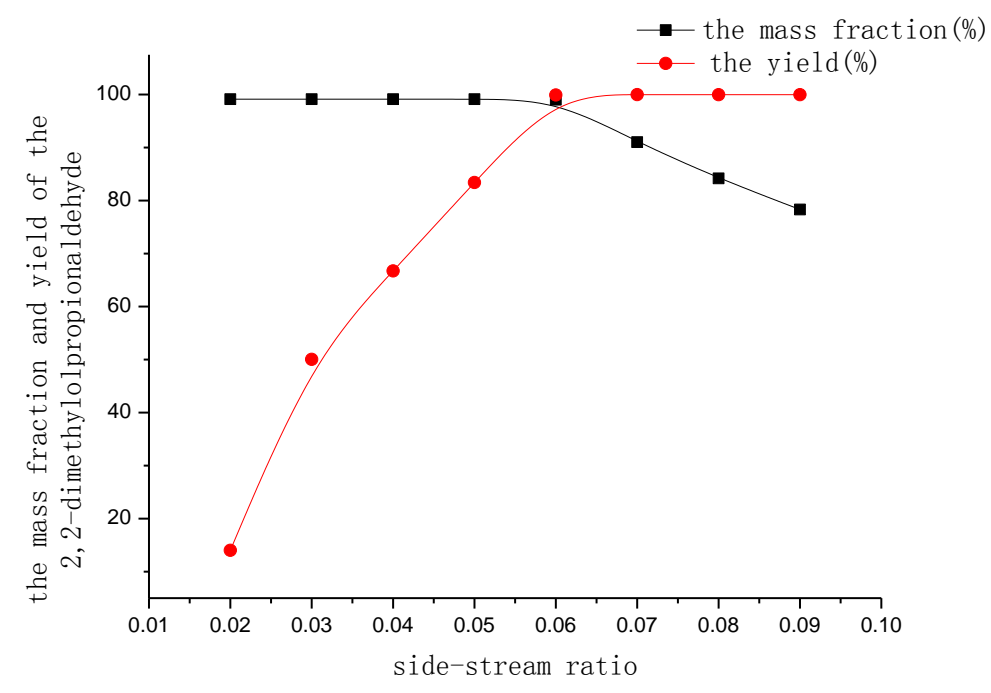

Fig. 6 The influence of the side-stream ratio

\section{Orthogonal Array Experimental Design}

In this study, the experiments are based on an orthogonal array experimental design $\left(\mathrm{L}_{16}\right.$ matrix) $[7,8]$ where the following five variable factors are analyzed: the theoretical stage number (factor A), feed stage (factor B), side-stream stage (factor C), reflux ratio (factor D), side-stream ratio (factor E). These variable factors are all identified to have large effects on the distillate mass fraction because the level of importance of each optimal condition is very different. A $L_{16}$ matrix, which is an orthogonal array of five factor and four levels, is employed to assign the considered factors and levels as shown in Tab. 1. 
Tab. 1 Levels and factors affecting the distillate mass fraction

\begin{tabular}{cccccc}
\hline \multirow{2}{*}{ Level } & \multicolumn{5}{c}{ Factors } \\
\cline { 2 - 6 } & $\begin{array}{c}\text { The theoretical } \\
\text { stage number A }\end{array}$ & $\begin{array}{c}\text { Feed stage } \\
\text { B }\end{array}$ & $\begin{array}{c}\text { Side-stream } \\
\text { stage C }\end{array}$ & $\begin{array}{c}\text { Reflux ratio } \\
\text { D }\end{array}$ & $\begin{array}{c}\text { Side-stream } \\
\text { ratio E }\end{array}$ \\
\hline 1 & 22 & 10 & 13 & 2.5 & 0.05 \\
2 & 24 & 11 & 15 & 3.0 & 0.06 \\
3 & 26 & 12 & 17 & 3.5 & 0.07 \\
4 & 28 & 13 & 19 & 4.0 & 0.08 \\
\hline
\end{tabular}

According to the $\mathrm{L}_{16}$ matrix, sixteen experiments are carried out and the distillate mass fraction results are shown in Table 2. This table shows that the range of the results varies from $83.75 \%$ to 99.28\%. What's more, the mean values of $\mathrm{K}$ for different factors at different levels in the range analysis are shown in the Table 3. And for each factor, a higher $\mathrm{K}$ indicates that the level has a larger influence on distillate mass fraction. So the best level for each factor can be determined according to the highest mean value of the experimental conditions. As we can see from the Table 3, the greatest mass fraction for each level is clearly distinguished, so the mean values of $\mathrm{K}$ is the highest at the combinations (A1B3C3D3E1). In addition, the range value indicates the importance of the factors' effect, and the larger $\mathrm{R}$ means that the factor has a greater effect on the distillate mass fraction[9]. Therefore, compared with the range values of the different factors, the factors' levels of significance are as follows, the side-stream ratio $(15.173)>$ side-stream stage $(2.210)>$ feed stage $(2.035)>$ the theoretical stage number $(1.843)>$ reflux ratio (1.671). It can be observed that the range $R_{E}$ is the largest, which means that the side-stream ratio has the most important effect on the distillate mass fraction and the distillate mass fraction changes slightly with changes in reflux ratio.

Tab. 2 Conditions and results of orthogonal experiment

\begin{tabular}{ccccccc}
\hline \multirow{2}{*}{ Number } & \multicolumn{7}{c}{ Factors } \\
\cline { 2 - 6 } & $\mathrm{A}$ & $\mathrm{B}$ & $\mathrm{C}$ & $\mathrm{D}$ & $\mathrm{E}$ & $\omega / \%$ \\
\hline 1 & $1(22)$ & $1(10)$ & $1(13)$ & $1(2.5)$ & $1(0.05)$ & 98.99 \\
2 & $1(22)$ & $2(11)$ & $2(15)$ & $2(3.0)$ & $2(0.06)$ & 98.82 \\
3 & $1(22)$ & $3(12)$ & $3(17)$ & $3(3.5)$ & $3(0.07)$ & 98.05 \\
4 & $1(22)$ & $4(13)$ & $4(19)$ & $4(4.0)$ & $4(0.08)$ & 83.79 \\
5 & $2(24)$ & $2(11)$ & $3(17)$ & $1(2.5)$ & $4(0.08)$ & 84.19 \\
6 & $2(24)$ & $1(10)$ & $4(19)$ & $2(3.0)$ & $3(0.07)$ & 90.94 \\
7 & $2(24)$ & $4(13)$ & $1(13)$ & $3(3.5)$ & $2(0.06)$ & 97.86 \\
8 & $2(24)$ & $3(12)$ & $2(15)$ & $4(4.0)$ & $1(0.05)$ & 99.28 \\
9 & $3(26)$ & $3(12)$ & $4(19)$ & $1(2.5)$ & $2(0.06)$ & 98.76 \\
10 & $3(26)$ & $4(13)$ & $3(17)$ & $2(3.0)$ & $1(0.05)$ & 99.12 \\
11 & $3(26)$ & $1(10)$ & $2(15)$ & $3(3.5)$ & $4(0.08)$ & 84.19 \\
12 & $3(26)$ & $2(11)$ & $1(13)$ & $4(4.0)$ & $3(0.07)$ & 90.98 \\
13 & $4(28)$ & $4(13)$ & $2(15)$ & $1(2.5)$ & $3(0.07)$ & 90.94 \\
14 & $4(28)$ & $3(12)$ & $1(13)$ & $2(3.0)$ & $4(0.08)$ & 83.75 \\
15 & $4(28)$ & $2(11)$ & $4(19)$ & $3(3.5)$ & $1(0.05)$ & 99.21 \\
16 & $4(28)$ & $1(10)$ & $3(17)$ & $4(4.0)$ & $2(0.06)$ & 99.06 \\
\hline
\end{tabular}

Tab. 3 Range analysis of results of orthogonal experiment.

\begin{tabular}{ccccccc}
\hline \multirow{2}{*}{} & \multirow{2}{*}{ Programs } & \multicolumn{5}{c}{ Factors } \\
\cline { 3 - 6 } The & K1 & A & B & C & D & E \\
\hline distillate & K2 & 94.910 & 93.295 & 92.895 & 93.220 & 99.150 \\
mass & K3 & 93.263 & 94.960 & 95,105 & 94.828 & 92.728 \\
fraction & K4 & 93.240 & 92.925 & 93.172 & 93.275 & 83.977 \\
& Range & 1.843 & 2.035 & 2.210 & 1.671 & 15.173 \\
& Optimization levels & A1 & B3 & C3 & D3 & E1 \\
& Optimal combination & & A1B3C3D3E1 & & \\
\hline
\end{tabular}

Then the distillation tower (T201) is also simulated by the same method using the Aspen Plus. 
The Table 4 shows the optimal operating conditions of the distillation tower.

Tab. 4 The optimal operating conditions of distillation tower.

\begin{tabular}{ccccc}
\hline equipment & $\begin{array}{c}\text { The theoretical } \\
\text { stage } \mathrm{N}\end{array}$ & $\begin{array}{c}\text { Feed stage } \\
\mathrm{N}_{\mathrm{f}}\end{array}$ & $\begin{array}{c}\text { Reflux ratio } \\
\mathrm{R}\end{array}$ & $\begin{array}{c}\text { Top discharge } \\
\text { ratio D/F }\end{array}$ \\
\hline $\mathrm{T} 201$ & 15 & 10 & 0.5 & 0.1504 \\
\hline
\end{tabular}

\section{Conclusions}

This study introduces a separation simulation of the mixture including 2,2-dimethylolpropionaldehyde, methanol, propylaldehyde, water and the high boiling organic compounds by continuous side-distillation and distillation using the Aspen Plus software. And the results of the simulation show that the method of separating the mixture is feasible by the NRTL model.

The each parameter is optimized by the sensitivity analysis in the simulation software and the orthogonal array experiment. The mass fraction and yield of the 2,2-dimethylolpropionaldehyde reach $99.01 \%$ and $99.91 \%$ respectively. And the total mass fraction of the methanol and propylaldehyde reaches $99.39 \%$ in the recovery tower, the yield of them reaches $99.96 \%$ and $99.99 \%$ respectively, meanwhile the mass fraction of the water reaches $99.99 \%$.

\section{References}

[1] Lijie Zhao, Study on catalytic synthesis of 2,2-dimethylolpropionaldehyde with phosphomolybdic acid, Chemical Engineer. Vol.2 (2006), p. 62-63.

[2] F.X.BALL, W.FURST, H.RENON, An NRTL Model for Representation and Prediction of Deviation from Ideality in Electrolyte Solution Compared to the Models of Chen (1982) and Pitzer (1973), AIChE Journal. Vol. 31 (1983), p. 392-393.

[3] Mehrdokht B. Nikoo, Simulation of biomass gasification in fluidized bed reactor using ASPEN PLUS, Biomass and Bioenergy. Vol. 32 (2008), p. 1245-1247.

[4] Shenfeng Yuan, Cancan Zou, Study on the separation of binary azeotropic mixtures by continuous extractive distillation, Chemical Engineering Research and Design. Vol. 93 (2015), p. 113-115.

[5] Songlin $\mathrm{Xu}$, Huiyuan Wang, A new entrainer for separation of tetrahydrofuran-water azeotropic mixture by extractive distillation, Chemical Engineering and Processing. Vol. 45 (2006), p. 955-956.

[6] Diana. Botia, Pablo Ortiz,Extractive Distillation of Acetone/Methanol Mixture Using Water as Entrainer, Ind. Eng. Chem. Res. Vol. 48 (2009), p. 4858-4864.

[7] Xuan Wu, Dennis Y. C. Leung, Optimization of biodiesel production from camelina oil using orthogonal experiment, Applied Energy. Vol. 88 (2011), p. 3615-3622.

[8] Yao.J.-Y, Lin, S,-J, Operation and control of batch extractive distillation for the separation of mixtures with minimum-boiling azeotrope, J.Chin.Inst.Chem.Eng. Vol. 38 ( 2007), p. 371-372.

[9] Brito.R.P., Optimal design of extractive distillation columns-a systematic procedure using a process simulator, Chem.Eng.Res.Des. Vol. 89 (2011), p. 341-344. 